\title{
A Fast Three-Dimensional Lighting Time Algorithm
}

Yao Jin

This article was submitted to

The $13^{\text {th }}$ American Physical Society Topical Conference on Shock Compression of Condensed Matter, Portland, OR, July $20^{\text {th }}-$ July $25^{\text {th }}, 2003$.

\section{July $14^{\text {th }}, 2003$}

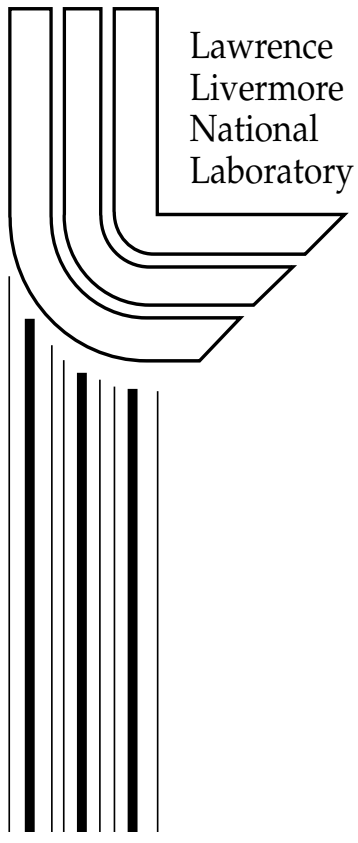




\section{DISCLAIMER}

This document was prepared as an account of work sponsored by an agency of the United States Government. Neither the United States Government nor the University of California nor any of their employees, makes any warranty, express or implied, or assumes any legal liability or responsibility for the accuracy, completeness, or usefulness of any information, apparatus, product, or process disclosed, or represents that its use would not infringe privately owned rights. Reference herein to any specific commercial product, process, or service by trade name, trademark, manufacturer, or otherwise, does not necessarily constitute or imply its endorsement, recommendation, or favoring by the United States Government or the University of California. The views and opinions of authors expressed herein do not necessarily state or reflect those of the United States Government or the University of California, and shall not be used for advertising or product endorsement purposes.

This is a preprint of a paper intended for publication in a journal or proceedings. Since changes may be made before publication, this preprint is made available with the understanding that it will not be cited or reproduced without the permission of the author.

This report has been reproduced directly from the best available copy.

Available electronically at http://www.doc.gov/bridge

Available for a processing fee to U.S. Department of Energy

And its contractors in paper from

U.S. Department of Energy

Office of Scientific and Technical Information

P.O. Box 62

Oak Ridge, TN 37831-0062

Telephone: (865) 576-8401

Facsimile: (865) 576-5728

E-mail: reports@adonis.osti.gov

Available for the sale to the public from

U.S. Department of Commerce

National Technical Information Service

5285 Port Royal Road

Springfield, VA 22161

Telephone: (800) 553-6847

Facsimile: (703) 605-6900

E-mail: orders@ntis.fedworld.gov

Online ordering: http://www.ntis.gov/ordering.htm

OR

Lawrence Livermore National Laboratory

Technical Information Department's Digital Library

http://www.llnl.gov/tid/Library.html 


\title{
A FAST THREE-DIMENSIONAL LIGHTING TIME ALGORITHM
}

\author{
Jin Yao \\ Lawrence Livermore National Laboratory \\ Livermore, California 94550.
}

\begin{abstract}
A narrow band level-set method to calculate the physical lighting time in three dimensions has been implemented with arbitrary hexahedral element systems.

This method does not involve finite difference calculation of spatial derivatives. Mesh regularity, and the local topologic equivalence to a regular mesh are not required. The lighting surface is represented by a collection of curved facets contained in partially burnt cells. Level-set functions are calculated by direct measurement of distance to the lighting surface, and are carried only for nodes of a set of elements in a narrow band that covers the lighting surface. In the case of a concave boundary, the nodal distances are calculated with geodesics. A polynomial fitting of nodal level-set values across elements provides third-order spatial accuracy where the surface is sufficiently smooth. The curvature of the lighting surface is explicitly calculated. The DSD levelset equation is integrated directly in the normal direction.
\end{abstract}

The new algorithm is self-initialized and allows easy boundary treatment with body fitting meshes. It can also be used as a fast, accurate solver for general surface propagation problems.

\section{INTRODUCTION}

Existing level-set lighting time algorithms for detonation propagation ${ }^{[1][2]}$ usually require mesh regularity, external initialization, and integration on all data points at every time step. These constraints make these algorithms inefficient with nontrivial boundary treatment, and of lower accuracy. The method presented in this paper is efficient, self-starting, of higher accuracy, and easily capable of treating general boundaries.

\section{NARROWBAND ALGORITHM}

The new method integrates the DSD level-set equation only for data points in a narrowband at a time step. For data points out of the narrow band, only the signs of their level-set functions are required (i.e. a point is burnt or not). The cost of numerical integration is one dimension lower than a full method. A narrowband method in two-dimensions can be easily implemented ${ }^{[3][4]}$. In comparison, a three-dimensional narrowband level-set method seems to require a significantly simplified approach because of the nontrivial connectivity of surfaces. The work described in this paper is the first complete description of a simple three-dimensional narrowband level-set method and its numerical implementation.

\section{BODY-FITTING MESH}

There is no requirement on mesh regularity with the new method. Without loss of generality, a finite element system is assumed to represent the numerical problem. The external boundaries and the material interfaces are assumed represented with cell faces. This is not required for the new method to work but by taking advantage of body-fitting meshes, the boundary treatment becomes trivial.

\section{ELEMENTARY APPROACH}

The new method is essentially an elementary approach based on straightforward distance calculations and least squares fitting. An outline of the procedures is as follows

1) Determine for each partially burnt cell a 'facet' using nodal level-set values, thereby constructing the lighting surface.

2) Calculate the signed normal distances to the surface for the nodes in a narrowband.

3) Integrate the DSD level-set equation in the normal direction for each narrowband node. 
Steps 1), 2), and 3) are repeated until all $\mathrm{HE}$ regions are burnt. The initial burn surface is assumed specified by the signed distances to the surface from the nodes of partially burnt cells.

\section{SELF-INITIALIZATION}

To start a full level-set calculation requires having the signed minimum distances from the entire set of data points to the initial burn surface. This is logically contradictory in the sense that initialization of such a problem is essentially equivalent to solving the entire problem. In the case of complex boundaries, initialization with signed minimum distances is nontrivial. In contrast, the new method only requires the calculation of signed minimum distances for points in a narrowband around the lighting surface at any given time. Since the narrowband data is sufficient to derive the solution, the new method is self-initialized, once the initial lighting surface is specified.

\section{SURFACE REPRESENTATION (FRONT CAPTURING TECHNIQUE)}

With the new method, the burn surfaces are represented with a collection of 'facets', which are contained in partially burnt cells. A facet is the portion of lighting surface cut off by a partially lighted cell. This concept can efficiently be used for general front capturing problems. Complexity of surface connectivity in three dimensions can then be handled with ease. A node that is owned by a partially burnt cell is considered as a surface node.

\section{LEVEL OF NEIGHBORS}

Level of neighbors is a powerful way to loop over a set of items that has connectivity of neighbor relations. It is an efficient approach for managing the facets on the lighting surface, as demonstrated in Figure 1.

\begin{tabular}{|l|l|l|l|l|l|l|}
\hline 3 & 3 & 3 & 3 & 3 & 3 & 3 \\
\hline 3 & 2 & 2 & 2 & 2 & 2 & 3 \\
\hline 3 & 2 & 1 & 1 & 1 & 2 & 3 \\
\hline 3 & 2 & 1 & 0 & 1 & 2 & 3 \\
\hline 3 & 2 & 1 & 1 & 1 & 2 & 3 \\
\hline 3 & 2 & 2 & 2 & 2 & 2 & 3 \\
\hline 3 & 3 & 3 & 3 & 3 & 3 & 3 \\
\hline
\end{tabular}

Figure 1. The level of neighbors.

\section{NARROWBAND}

The narrowband is defined as the set of all the surface nodes (which define all the partially lighted cells) and the nodes of the first level of unburned neighbor cells of partially burnt cells. Among these nodes, the surface nodes are used to determine the burn surface at the current time with their level-set values. The rest will have their level-set values calculated at the current time step, and are going to define the burn surface at later time steps as they have become new surface nodes. Clearly, a thinner narrowband provides more efficiency. The definition of narrowband here makes it the thinnest possible thus the method is optimized.

\section{CHARACTERISTIC LENGTH}

Any node in the narrowband cannot possibly have a distance to the region of partially burnt cells greater than the maximum cell dimension (the diameter of a smallest sphere that may contain any cell). The maximum cell dimension is the characteristic length with this method.

\section{FACET}

The vertices of a facet are first determined with a linear interpolation of nodal level-set values on the edges of a given partially burnt cell. Such an approximation can exactly propagate a planar wave. These vertices are not necessarily on a plane. We fit the facet with a plane to define a local Cartesian coordinate for an initial nodal distance calculation. This facet-coordinate has its origin at the vertical projection of a node along an axis that coincides with the facet normal.

\section{NODAL DISTANCE}

The nodal distance is defined as the shortest path from a node to a facet. The distance from a given narrow band node to the surface is the minimum among the distances measured from the node to all facets. The facet corresponding to the shortest path will be used to refine the initial nodal distance calculation in its facet-coordinate.

\section{REGION OF INFLUENCE}

The region that contains all points with a distance less or equal to the characteristic length (the maximum cell dimension) to a given cell is defined as the "region of influence" of this cell. For a given facet, we only need to calculate for the nodes inside the region of influence and inside the narrowband. Since the information for these nodes is sufficient to propagate the surface, all of the other nodes can be eliminated from the calculations of the nodal distance to this facet. 


\section{POLYNOMIAL SURFACE FITTING}

To more accurately calculate the nodal distance, on the smooth portion of the burn surface, one can fit the surface with a quadratic polynomial of two variables in the facet-coordinate when curvature is small, using known surface nodal level-set values. This gives the third order of accuracy required for the calculation of the curvature.

\section{LEAST SQUARES FITTING AND FINITE DIFFERENCE METHODS}

The polynomial fitting is done by selecting a set of surface nodes in the region of influence of a facet and by fitting their level-set values with a least squares method. It is worth noting that general finite difference methods are strictly equivalent to special cases of the least squares method as used here ${ }^{[5]}$. Compared to finite difference methods, the new algorithm is much more flexible.

\section{CURVATURE}

The integration of the DSD level-set equation requires an explicit curvature calculation. This calculation is tedious, even on regular meshes with finite difference methods. A second order method does not provide the required Taylor expansion terms to obtain curvature. Thus a second order method that claims to have curvature effect is not self-consistent. The third order of accuracy of the new method is necessary and sufficient for the curvature term to be included in the calculation. The calculation of curvature is trivial with the quadratic surface fitting polynomial.

\section{INTEGRATING ON THE NORMAL}

The DSD level-set equation is expressed as

$$
\frac{\partial \varphi}{\partial t}+D_{n}(\kappa)|\nabla \varphi|=0,
$$

for a quasi-steady detonation, here $\varphi$ is the level-set function, $D_{n}$ is the normal detonation speed and $\kappa$ is the curvature. With a finite difference method, the curvature and the norm of gradient cannot be calculated in simple ways. However, on the surface normal, the DSD levelset equation reduces to

$$
\frac{d \varphi}{d t}+D_{n}(\kappa)=0
$$

This is much easier to solve using the new method since the surface normal vector and curvature of the burn surface are naturally obtained. The integration of the DSD level-set equation can be done trivially in the normal direction. If the detonation velocity is a constant, one simply has $\phi(t+d t)=\phi(t)-D_{n}(d t)$, which is the same as a simple Huygens construction.

\section{TIME STEP}

The time step is determined by the minimum time it takes for the current surface to reach a non-surface node in the narrowband. This means the lighting surface at the next time step is completely determined by information in the current narrowband. A factor less than the unity can be used to further limit the time step.

\section{ASPECT RATIO}

Naturally, the new algorithm can be performed on a regular mesh. It is most effective when the cells are cubic.

For non-cubic cells, the time step of this method may be reduced when the detonation wave is traveling parallel to the short cell dimension. In addition, a curved burn front can light the center of a given cell without any of its nodes being lit. This situation may cause a few facets to be missing from the lighting surface. Although polynomial fitting of the surface tends to have the missing portion reconstructed, particularly thin cells should be avoided if possible.

\section{MULTIPLE DETONATION FRONTS}

Lighting surfaces are considered as collections of facets. On the facet level, the case of multiple lighting surfaces and the case of single lighting surface are not treated differently with the new method.

Let us consider the case of interactions between multiple lighting fronts in some detail. In theory, it is possible for a partially burnt cell to contain several pieces of lighting surfaces. It looks like a complicated situation. In practice, the solution is simple with the narrowband approach. We argue that a) a cell in which such a complex situation occurs is not going to be used to generate a facet for determining nodal distances of other narrowband nodes, and b) the nodal distances in this cell are determined in some previous time steps. The accuracy of the new method is therefore not affected by multiple front interactions.

\section{MATERIAL INTERFACES}

A detonation front is assumed to intersect material interfaces with determined angles. With the assumption of body-fitting mesh, a facet in a boundary cell can be determined with the angle 
between the facet and the cell walls. This makes the boundary condition trivial to apply.

If the interface is between two HE materials of different detonation velocities, the law of refraction is taken into account in determining the nodal distance.

\section{GEODESICS}

Since a given facet needs only to be considered within its region of influence, the new method can treat complex boundary geometry in an easy fashion. When the regions of $\mathrm{HE}$ material have concave boundary portions, the path of minimum lighting time may not be determined by a straight line. To compute the shortest path between two points, one does a geodesic calculation on the plane that contains the principal surface normal and the points where the boundary is smooth. It can be easily shown that such an approximation is $3^{\text {rd }}$ order accurate. If sharp concave edges are present in the region of influence, one may use a plumb line to determine the nodal distance.

\section{LIGHTING TIME ON CELL CENTERS}

Nodal average can give at most $2^{\text {nd }}$ order of accuracy for the lighting time at cell centers. If computing cost is not a burden, one should treat cell centers as nodes and carry level-set values on them, as well. An alternative is to use high order MLS interpolation of nodal values.

\section{NUMERICAL EXPERIMENT}

A cubic of size 40 by 40 by 40 is ignited at corner $(20,20,20)$ and $(-20,-20,-20)$ at time zero. The lighting velocity is set to 1 . The initial burn surfaces are two $1 / 8$ spheres with a radius of 10 thus about $1.64 \%$ of the total volume is burnt when the calculation starts. 4 different cell sizes are tested. The non-dimensional nodal errors of lighting time compared to theoretical solution are the follows (the amounts inside parentheses are for narrowband nodal distances measured at the first time step).

\begin{tabular}{|l|c|c|c|c|}
\hline CELL SIZE & $4 \times 4 \times 4$ & $2 \times 2 \times 2$ & $1 \times 1 \times 1$ & $2 \times 2 \times 1 / 2$ \\
\hline L2 ERROR & $\begin{array}{c}.002158 \\
(.002078)\end{array}$ & $\begin{array}{l}.000118 \\
(.00006)\end{array}$ & $\begin{array}{l}.000028 \\
(.000008)\end{array}$ & $\begin{array}{l}.000198 \\
(.000175)\end{array}$ \\
\hline MAX ERROR & $\begin{array}{c}.009588 \\
(.00958)\end{array}$ & $\begin{array}{c}.000410 \\
(.000385)\end{array}$ & $\begin{array}{c}.000088 \\
(.000053)\end{array}$ & $\begin{array}{c}.012225 \\
(.002008)\end{array}$ \\
\hline TIME STEPS & 9 & 18 & 35 & 64 \\
\hline
\end{tabular}

Figure 2. The nonlinear convergence.
It is easy to see the high accuracy, and the nonlinear convergence as the cell size decreases. The case of big aspect ratio introduces a relatively large maximum local error (where curvature is big) but the $L_{2}$ error stays small.

\section{CONCLUSIONS}

The narrowband nature of the new threedimensional lighting algorithm makes it a fast one. The concept of region of influence has simplified the full problem to a collection of simple geometry problems. Direct distance calculations and least squares fitting are basically all that are needed for the method to work. The high order of accuracy and the low order of computing cost by the new method can probably make it a preferred method to solve general surface propagation problems.

\section{ACKNOWLEDGMENT}

This work has been developed from scratch since summer of 2002 with encouragement and direction from Tom Adams and Richard Sharp of Lawrence Livermore National Laboratory. The work is also benefited from my working experience with Scott Stewart at the University of Illinois at Urbana-Champaign and Dan Matuska at General Dynamics Ordinance and Tactical Systems at Niceville, Florida. The author also appreciates discussions with John Bdzil of Los Alamos National Laboratory.

\section{REFRENCES}

[1]. Level Set Methods Applied to Modeling Detonation Shock Dynamics, Tariq D. Aslam, John B. Bdzil, and D. Scott Stewart, JCP. 126, pp. 390-409, 1996.

[2]. The Level Set Method Applied to Threedimensional Detonation Wave Propagation, S. Wen, C. Sun, F. Zhao, and J. Chen, The Twelfth International Detonation Symposium, 2002.

[3]. A Fast Level Set Approach for Propagating Interfaces, D. Adalsteinsson, and J. A. Sethian, JCP. 118, pp. 269-277, 1995.

[4]. Level Set Methods and Fast Marching Methods, J. A. Sethian, Cambridge University Press, 1999.

[5]. Simulation of Detonation Problems with the MLS Grid-Free Methodology, J. Yao, M. E. Gunger, and D. A. Matuska, The Twelfth International Detonation Symposium, 2002. 
